\title{
Dimensión político-ideológica de la discapacidad: Un análisis desde el enfoque socio-crítico
}

\author{
Political-ideological Dimension of Disability: \\ An Analysis from a Socio-critical Approach
}

\author{
Marie Claire Vargas Dengo' \\ División de Educación Básica \\ Centro de Investigación y Docencia en Educación \\ Universidad Nacional \\ Heredia, Costa Rica \\ educare@una.ac.cr
}

\begin{abstract}
Recibido 10 de noviembre de 2011 • Corregido 22 de mayo de 2012 • Aceptado 28 de junio de 2012
Resumen. Desde el enfoque socio-crítico, se analiza la dimensión política e ideológica de la discapacidad con el fin de identificar procesos estructurales subyacentes a las políticas educativas en materia de discapacidad. El artículo contrasta las ideas y posiciones de distintos autores, y expone la vigencia y relevancia del tema para promover la inclusión educativa en la educación superior.
\end{abstract}

Palabras claves. Discapacidad, enfoque socio-crítico, inclusión educativa, educación superior.

Abstract. This paper analyzes the political and ideological dimensions of disability, from a sociocritical approach, in order to identify key structural processes underlying the educational policies concerning disability. The paper compares the ideas and approaches of various authors, and points out the relevance of the topic to promote inclusive education in higher education.

Keywords. Disability, socio-critical approach, inclusive education, higher education.

\section{Introducción}

El paradigma ideológico dominante en una sociedad se refleja en las concepciones, las formas de vida, las prácticas sociales, educativas y laborales vigentes en un determinado momento histórico. Los cambios ideológicos repercuten en dichas prácticas, así como en las maneras de pensar y en las concepciones, lo cual se refleja en las formas de vida, en el trato social, en la educación y en lo laboral.

Máster en Currículum e Instrucción con énfasis en Educación Especial Bilingüe de The George Washington University, USA; Doctoranda de la VIII promoción del Programa Latinoamericano de Educación de la Universidad de Costa Rica. Académica de la División de Educación Básica del Centro de Investigación en Docencia y Educación (CIDE) de la Universidad Nacional (UNA) en Costa Rica. Integró por varios años el Proyecto UNA educación de calidad, el cual atiende al estudiantado con discapacidad en dicha universidad. 
La importancia de ubicar histórica, cultural y contextualmente distintos paradigmas se debe a que, de una u otra forma, todos ellos reflejan prácticas sociales dominantes y legitimadas, en diversas etapas históricas. Desde esta perspectiva, el enfoque socio-crítico aborda el tema de la discapacidad para explicar las implicaciones ideológicas y las repercusiones sociales, las cuales se reflejan claramente en la discrepancia entre dos modelos de la discapacidad: el modelo individual y el modelo social. Si bien desde algunas perspectivas críticas se cuestiona el discurso de la inclusión educativa, la inclusión se delinea como una tendencia actual y generalizada en distintos espacios sociales, educativos y laborales.

\section{Inclusión educativa: una tendencia actual}

En términos generales, la temática de la inclusión ha sido abordada desde dos grandes líneas, una de ellas es más amplia y se refiere a la inclusión social; mientras que la otra, más específica, se circunscribe a los entornos educativos y, por tanto, se le denomina inclusión educativa.

La inclusión social apunta a permitir mayores oportunidades de participación social, especialmente, a las personas de grupos minoritarios que han sido marginadas en la sociedad por razones de cultura, etnia, género, discapacidad, entre otros (Hanafin, Shevlin, Kenny y Mc Neela, 2007). Concebir la inclusión social como un derecho fundamental en una sociedad civilizada significa permitir que, a partir de su experiencia, todas las personas puedan contribuir a la vida pública (Skelton, 2002). La inclusión educativa apela a ampliar la concepción tradicional de la pedagogía y se define como el derecho a la educación independientemente de las diferencias y disposiciones individuales.

Con el fin de mitigar las situaciones de exclusión a las que estaban siendo sometidos muchos estudiantes con necesidades educativas especiales y con discapacidad, el movimiento inclusionista educativo se inicia a finales del siglo XX, desde el campo disciplinar de la Educación Especial y es posterior al movimiento de integración (Arnaiz, 2010).

La inclusión educativa, actualmente, se propone en términos de responsabilidad compartida, ya que busca el cambio en los sistemas educativos con el fin de responder a la diversidad del estudiantado y garantizarle un trato igualitario. Este constituye un proceso que compromete a toda la sociedad (Arnaiz, 2010) y, aún más, a quienes conforman las comunidades de las distintas instituciones educativas. Dado que la inclusión educativa no está dada de previo, su construcción requiere indispensablemente fomentar una cultura institucional inclusiva, lo cual implica fortalecer los valores que sustentan la equidad, la diversidad y la justicia social (Moran, 2007).

En el contexto de la educación superior, las instituciones universitarias pueden servir como instrumento integrador, en tanto ofrezcan igualdad para todas las personas en el acceso a la educación (Skelton, 2002). Al promover la inclusión educativa en términos de participación 
y acceso al conocimiento en los distintos espacios institucionales, contribuirían aún más con la función social que desempeñan. Sin embargo, esto demanda una democratización del conocimiento que fortalezca la autonomía y la libertad personal, así como un cambio de mentalidad y actitudes hacia las diferencias individuales en los distintos espacios institucionales específicos en que se desarrolla la educación superior. De acuerdo con Barnes (2007b), aún hay un largo camino que recorrer para que el sistema de educación superior sea realmente inclusivo. Según dicho autor, una verdadera educación inclusiva, implicaría que la educación superior respondiera al nivel de desempeño y capacidades individuales independientemente de las particularidades que las personas presenten.

\section{Paradigmas ideológicos y discapacidad: el enfoque socio-crítico}

En materia de discapacidad, en las diferentes sociedades han predominado distintos paradigmas en distintas épocas, de tal forma que "(...) los contextos históricos contribuyen a configurar lo que ahora damos en llamar discapacidades" (Martínez, s. f., p. 3). Las concepciones y, por tanto, las prácticas sociales hacia las personas con discapacidad, sin duda alguna, han cambiado paulatinamente.

Desde la perspectiva materialista social, Oliver (1990) ubica históricamente la discapacidad dentro del auge de la sociedad capitalista. Sostiene que lo que determinó la concepción de la discapacidad como un problema individual, médico y de tragedia personal fue el cambio a la sociedad capitalista, ya que la sociedad capitalista se rige por intereses empresariales y por la economía de mercado. En este tipo de sociedad, la productividad requiere de trabajadores útiles, eficientes y capaces; no así de las personas con discapacidad, consideradas no óptimas para lograr tales propósitos.

Oliver (1996) argumenta que debido a que las condiciones de la producción capitalista cambiaron en el siglo XX, "(...) la necesidad de mano de obra giró de la masificación de trabajadores sin habilidades a una necesidad más limitada de trabajadores con habilidades (...)" (Manning y Oliver, 1985, p. 102). Como resultante, al emerger el Estado de Bienestar, este procura proveer una mano de obra con las destrezas necesarias, a la vez que asegura pacificar a los desempleados y a los no empleables.

La sociedad capitalista se caracteriza por ser de mercado regulado, a la vez que es regulada por el mercado. Su modelo histórico, económico e ideológico promulga la ideología liberal-individualista. Esta concepción de individualismo es lo que Oliver (1996) retoma y refiere como 'la hegemonía del modelo individual' de la discapacidad, para destacar su dominancia y prevalencia histórica. Además, acota que, si bien tal hegemonía ha sido desafiada por el modelo social, no ha logrado sustituirlo completamente.

En esta misma línea, Ferreira (2007, p. 5) coincide con Oliver (1990) al señalar que "(...) la condición de discapacitado sería consecuencia del surgimiento de la fuerza de trabajo industrial. 
La discapacidad surgiría como categoría excluida de esta nueva clase social (...)". Desde este enfoque, la exclusión de las personas con discapacidad del mundo laboral se debe a los intereses productivos, mientras que la exclusión social adquiere distintas manifestaciones como lo son el aislamiento y la segregación en instituciones apartadas de la sociedad. Por lo cual, las prácticas de aislamiento y de institucionalización de las personas con discapacidad se fomentan con vigor a inicios de la época de la industrialización. Con ello se incrementan las prácticas asistenciales de cuido, reclusión y tratamiento individual, lo cual, a la vez, promueve la formación de profesionales especialistas para el cuido, educación y tratamiento de las personas con distintas discapacidades. El tratamiento adquiere un carácter rehabilitador y médico, centrado en el déficit, es decir, en la deficiencia personal y en la incapacidad de desempeño individual. De acuerdo con el enfoque médico, las personas con discapacidad son poseedoras de cuerpos no legítimos según la norma médica, por lo que tendrán que basar sus expectativas en la obtención de una legitimación en segunda instancia, una falsa legitimación, o bien, la legitimación de lo ilegítimo (Ferrante y Ferreira, 2011). Según Ferreira (2007), sobre estos criterios se ha elaborado a lo largo de dos siglos, la categoría 'social' de la discapacidad, la cual "(...) implica un individuo carente y no funcional, que padece una insuficiencia, que está destinado a la caridad y cuyo mejor destino es la cura (...)" (p. 6).

Lo anterior da origen al proteccionismo asistencial que posteriormente se ve fortalecido con las políticas del 'beneficio' proteccionista promulgadas por el Estado Benefactor en procura del cuido, el tratamiento y la cura. En estos términos, el Estado de Bienestar enmascara, impulsa y perpetúa un modelo individualista de la discapacidad, en tanto que "(...) expresan su razón de ser: el mercado, la lógica del beneficio, la primacía del interés individual (...)" (Ferrante y Ferreira, 2011, p. 90). Aunado a esto, la visión liberal sostiene que la desigualdad es resultante de la repartición de capacidades y merecimientos individuales en tanto que "(...) sólo aquéllos que poseen suficiente talento podrán optar a los lugares más privilegiados de la pirámide social: sus aptitudes y capacidades, si se esfuerzan en desarrollarlas adecuadamente, les harán merecedores de tales posiciones y, a cambio, obtendrán el reconocimiento general (...)" (Ferreira y Díaz, 2007, p. 2). Según estos autores, la visión liberal-meritocrática explica la ubicación de los individuos según sus capacidades y esfuerzo dentro de la jerarquía social.

\section{Implicaciones ideológicas y sociales de la discapacidad: el modelo social versus el modelo individual}

En la segunda mitad del siglo XX, como resultante de los movimientos activistas de lucha por los derechos humanos, se promulgan leyes y declaraciones en distintos países: las personas con discapacidad pasan a ser sujetos de derechos. En el campo educativo, se procuró la integración de estudiantes con discapacidad en instituciones educativas públicas y en aulas regulares, así como, posteriormente, se propició la provisión de apoyos pedagógicos para atender las necesidades educativas. En materia legislativa, el movimiento social de las personas 
con discapacidad promovió una legislación garante de sus derechos. Sin embargo, todavía hoy prevalecen concepciones de la discapacidad que limitan el trato equitativo y no excluyente.

El paso de un paradigma a otro en el último tercio del siglo XX ha propiciado reducir las barreras del entorno, como también hacer cambios arquitectónicos en procura de mejores condiciones de acceso al espacio físico. Este cambio de paradigma se evidencia, claramente, en las condiciones de accesibilidad tanto al espacio físico como en los espacios educativos y laborales.

Además, el paso de un modelo a otro ha significado no solo un cambio de perspectiva sino también de resignificación de la discapacidad como problemática social y política. Se ha cambiado la mirada sobre el tema pasando de considerarlo un problema individual, a identificarlo como una problemática de dimensiones políticas, implicaciones y consecuencias sociales. No es sino hasta cuando emerge el modelo social en contraposición al modelo individual y que, activistas y académicos desde la sociología crítica profundizan en el tema, que se reconocen las luchas sociales de los movimientos de las personas con discapacidad como luchas políticas, se cuenta con información válida para la toma de decisiones y políticas en materia de discapacidad, y se logra rescatar y plasmar la vivencia de la discapacidad desde la mirada de las personas con discapacidad (Oliver, 2002).

Al cobrar vigencia el modelo social de la discapacidad, esta se define en términos de la interacción de la persona con el entorno, lo cual implica que es el entorno lo que limita a las personas, en consecuencia, se magnifican las condiciones de discapacidad debido a las barreras que este impone. Esta concepción sustituye la visión de que la discapacidad es una característica o atributo personal, ya que concibe la discapacidad en una dimensión relacional con el entorno y no en una dimensión exclusivamente personal. De acuerdo con Barnes (2007a), en el modelo social de la discapacidad se destacan tres aspectos claves, como se explica a continuación.

En primer lugar, el modelo social contrasta con el modelo individual, de carácter médico, en tanto que gira el foco de atención de las limitaciones funcionales individuales a las barreras culturales y del entorno. En segundo lugar, el enfoque del modelo social se caracteriza por ser holístico, dado que explica las problemáticas vividas por las personas con discapacidad en relación con la totalidad de los entornos y de las culturas 'discapacitantes'. En tercer lugar, la perspectiva social no niega la importancia de las intervenciones individuales -sean médicas o rehabilitadoras, educativas o laborales-, pero sí centra la atención en el empoderamiento y la inclusión en una sociedad que ha sido construida por personas 'sin discapacidad' para personas 'sin discapacidad' (Barnes, 2007a).

Los aspectos mencionados evidencian el énfasis de la perspectiva del modelo social en contraste con el modelo médico individual, en términos de las barreras del entorno -sean estas físicas; de acceso a la información y comunicación, al transporte; culturales; educativas, o bien laborales-. Estas barreras, en todos los casos, implican la imposición de dificultades y obstáculos a la persona con discapacidad para desenvolverse en equidad de condiciones y situaciones que el resto de las personas sin discapacidad, lo cual implica una situación de 
diferencia y de desventaja. El modelo social de la discapacidad no pretende convertirse en una teoría social, como tampoco constituirse en la versión histórico-materialista de la discapacidad que dé explicación a las fallas del Estado de Bienestar con respecto a la provisión de servicios para las personas con discapacidad. Este modelo aporta un marco que posibilita comprender las tendencias de la sociedad moderna y la importancia de generar políticas y legislación que repercutan en las prácticas sociales (Barnes, 2007a). Desde la perspectiva de Oliver (1996), el modelo social de la discapacidad apela a las experiencias personales y colectivas y cómo las barreras sociales pueden influenciar la práctica profesional y conformar la acción política.

El modelo social de la discapacidad ha sido llamado 'la gran idea' del movimiento británico de la discapacidad (Shakespeare, 1993), movimiento desarrollado en los años setenta por los activistas de la Union of the Physically Impaired Against Segregation (UPIAS). El modelo social de la discapacidad ha tenido gran impacto en Gran Bretaña por cuanto facilitó identificar una estrategia política a partir de la eliminación de barreras para propiciar la inclusión social, de manera que se alejó de la consideración de la rehabilitación y la cura como única solución. En el ámbito académico logró auge y credibilidad, gracias a los trabajos de Finkelstein (1980), Barnes (2001), Oliver $(1990,1996)$ y Shakespeare (1993) y, si bien se retoma en distintas teorías para explicar la discapacidad como construcción social, está asociado al enfoque materialista (Barnes, 2007a). De acuerdo con Shakespeare (1993), desde esta postura se argumenta que las personas con discapacidad son un grupo social oprimido y se define la discapacidad en términos de opresión social y no de deficiencia individual. A diferencia de la perspectiva de los teóricos norteamericanos, quienes también han desarrollado un enfoque social como aproximación a la temática, sin la definición de discapacidad en términos de opresión social, el enfoque británico sí lo logra.

Autores como Barnes (1998) han criticado la bibliografía norteamericana proveniente de Estados Unidos y Canadá que aborda esta temática, pues argumentan que, de alguna forma, se ignora el trabajo de investigadores británicos en esta línea, no se adhieren a la definición de discapacidad que propone el modelo social y todavía se refieren a la discapacidad en términos biológicos y funcionales. Oliver (1996), por ejemplo, plantea que el asunto tiene implicaciones tanto biológicas como sociales; concuerda con Barnes (2007a) en que no se trata solamente de un asunto 'políticamente correcto', sino que es un hecho de causalidad, del sentido del uso del lenguaje y de sus tendencias 'normalizadoras', así como de la politización misma del proceso de definición. Estos argumentos concuerdan con los de Brogna (2005), quien sostiene que:

(...) El significado de la diferencia es un terreno de batalla política y que la discapacidad es situacional, relacional e interactiva con los factores ambientales, actitudinales y culturales: (...) La identidad "discapacitada" es asignada desde grupos significativos de poder, especialmente salud y educación, en relación a una deficiencia (física, mental o intelectual) a través de procesos de categorización, diagnóstico y etiquetamiento. (p. 46) 
En la misma línea, otros autores como Finkelstein (1980) sostienen que la discapacidad no es un atributo personal, sino que devela una relación social opresiva entre las personas con deficiencias físicas y la sociedad. Concuerdan con esta posición Ferrante y Ferreira (2008), quienes retoman las propuestas teóricas en la línea de Bourdieu (1991), las cuales sostienen que en las sociedades capitalistas la posición de un agente en el espacio social está determinada por su trayectoria social, así como por sus propiedades corporales, sean estas socialmente elogiadas o estigmatizadas. En este sentido, se alude a una relación de dominación y de poder de una clase dominante sobre un grupo minoritario oprimido. Esta concepción es ilustrada por Ferrante (2007), cuando distingue dos caras de la discapacidad -una objetiva y otra subjetiva-, al referirse a sus dos dimensiones: la dimensión 'situación de discapacidad' que refiere determinadas condiciones generales de la discapacidad como atributos objetivos en un contexto particular y, la dimensión 'condición de discapacidad' que refiere la experiencia de esas condiciones generales objetivas en términos individuales y subjetivos de la persona. Además de caracterizar esta relación social como opresiva, Ferreira y Díaz (2007) califican la experiencia de la discapacidad en términos de exclusión social, en la que tanto personas como instituciones y legislación han sido partícipes, lo cual contribuye a caracterizar a la sociedad como excluyente.

\section{Aspectos sociopolíticos e ideológicos subyacentes a las políticas educativas en materia de discapacidad}

Las disposiciones legislativas internacionales en materia de discapacidad han incidido en el tratamiento y discusión de esta temática en la educación superior tanto en el ámbito nacional como internacional, específicamente, desde la década de los años noventa (Barnes, 2007; Bolt, 2004; Lavia, 2007; Moran, 2007; Pumfrey, 2008 y Williams, 2006). Las políticas nacionales e internacionales en materia de discapacidad pretenden garantizar los derechos de las personas con discapacidad en distintos ámbitos de su vida, sean estos laborales, educativos, sociales o recreativos, entre otros; así como garantizar que a las personas con discapacidad se les reconozca como personas-ciudadanas y sujetos de derecho. Sin embargo, si bien existe un marco normativo para garantizar los derechos de las personas con discapacidad, en la práctica muchas normativas no se aplican con agilidad, eficacia y rigor (Ferreira y Díaz, 2009); en muchos casos esto se debe a que el cambio de concepciones y formas de pensar lleva más tiempo y, por tanto, se da un desfase entre el marco legal-normativo y las prácticas sociales y, lógicamente, las institucionales.

Los conceptos ideológicos claves en el pensamiento educativo moderno están permeados por un conjunto de relaciones particulares que caracterizan la enseñanza legitimada en la que el Estado cumple un papel regulador social al promulgar cierto tipo de legislación. Muchas políticas en materia de discapacidad han cobrado vigencia internacional a partir de las luchas sociales de los grupos minoritarios. Esto ha incidido en que, en la actualidad, la temática de la discapacidad sea 'políticamente correcta' y por tanto amerite estar presente en las agendas políticas de distintos países. En este sentido, los planteamientos de Popkewit (1997) facilitan 
URL: http://www.una.ac.cr/educare

la comprensión de cómo las propuestas de políticas subyacen al cambio educativo y, a la vez, pautan las prácticas sociales. De ahí que sea relevante develar la ideología subyacente en las políticas educativas vigentes en un período socio-histórico particular, como se plantea a continuación.

\section{Legislación costarricense en materia de discapacidad}

En el contexto político costarricense, las leyes y las políticas existentes respecto a la temática de discapacidad se aprobaron, principalmente, en las décadas de los años 1990, 2000 y 2010 (recientemente en el 2011 se aprobaron las Políticas Nacionales en materia de Discapacidad). En las décadas mencionadas, sin embargo, la materia social no ha sido prioridad de las políticas públicas del Estado, sino que, por el contrario, se caracterizaron por la liberalización económica iniciada a partir de la crisis de la década de los ochenta, periodo en el que prima el desmantelamiento del Estado de Bienestar y en el que se propulsan las tendencias de corte neoliberal, opuestas a que el Estado continúe brindando servicios sociales. Por el contrario, se promueve la privatización de los servicios y de las instituciones públicas, así como la economía de libre mercado y los intereses empresariales.

El deterioro del Estado de Bienestar causa desprotección social, la cual se agrava con las medidas políticas de corte neoliberal impuestas a países en vías de desarrollo, como es el caso de Costa Rica. En concordancia con los planteamientos de Riutort (2001), el poco compromiso del Estado con la sociedad y la reducción de políticas sociales caracterizan el capitalismo desregulado, así como también los mercados globalizados y flexibles, la privatización de las empresas estatales, menos empleos para los trabajadores y las nuevas formas de determinar los impuestos.

En este contexto, prevalece el interés privado y una racionalidad política legitimadora de la desigualdad social, contexto en el cual el capitalismo de mercado económico global logra establecer su hegemonía ideológica y cultural y, por medio de disposiciones educativas, legitimar el programa político de corte neoliberal (Miranda, 2004). Esta descripción coincide con el análisis de Apple (1997), cuando se refiere a la alianza gestada entre el neoconservadurismo y el neoliberalismo. Sostiene que esta acarrea, como consecuencia, la transformación de las condiciones económicas debidas al asentimiento de la economía neoconservadora, en la cual el conservadurismo impone sus prácticas sociales y políticas. Esto se refleja en imposiciones y demandas de las empresas e industrias privadas y no son otra cosa que condiciones impuestas para el logro de sus intereses y objetivos económicos, aún cuando estos aparezcan revestidos de una imagen de bienestar social.

A la luz de los verdaderos intereses económicos subyacentes, resulta perentorio develar este enmascaramiento de las políticas de corte social, pues distan de responder a las demandas sociales reales, existentes mundialmente, muchas de las cuales apelan a los derechos humanos. Sin embargo, el tema de los derechos humanos en la agenda política mundial adquiere otras 
dimensiones de análisis desde la óptica sociológica y estructural. Al respecto, plantea Sojo (2010, p. 101) que "la expansión universal de los derechos en tiempos de globalización es una oportunidad para las políticas igualitaristas (...)". En estos términos, la desigualdad es una herramienta política e ideológica que sustenta los discursos políticos oportunistas. Es decir, una bandera política de la cual los grupos de poder toman ventaja para que la ciudadanía luche por un ideal promisorio de igualdad "(...) una aspiración acotada de gobierno de la diferencia en procura de la igualdad" (Sojo, 2010, p. 110), en tanto que difunde un 'discurso utópico' de éxito y oportunidades sociales, un 'horizonte de bien común', el cual tiene un trasfondo estructural que reviste diferencias económicas de base, que revelan la idea de la desigualdad contenida en la ideología dominante que se remonta a las épocas de la constitución de la República costarricense y se prolonga hasta los fines del siglo XIX en que se construye "(...) un referente igualitarista para la definición de la nación y la sociedad costarricense (...)" (Sojo, 2010, p.166).

Desde la perspectiva anteriormente expuesta, la llamada 'igualdad de oportunidades' adquiere, entonces, un matiz político discursivo, a expensas de la expansión de los derechos en épocas de la globalización. Esto podría utilizarse para explicar por qué, si bien existen políticas y legislación en materia de discapacidad, las prácticas sociales no son coherentes con lo que se acepta como políticamente correcto. Pues si bien las concepciones hacia las personas con discapacidad han cambiado paulatinamente, aún prevalecen muchas prácticas sociales estancadas en el pasado. Cabe subrayar que el asunto no se trata únicamente de accesibilidad y de cambiar el entorno: se requieren cambios ideológicos que se manifiesten en las formas de pensar y actuar, y se evidencien en los distintos espacios sociales, educativos y laborales.

Desde algunas perspectivas críticas, se cuestiona si las prácticas inclusivas son un instrumento de competitividad y de estrategia de mercado para promocionar una imagen de educación abierta a las diferencias. Desde la sociología crítica, por ejemplo, se argumenta que la igualdad de oportunidades y la diversidad son discursos políticos propios del modelo economicista de la educación (Sheeran, Brown, y Baker, 2007) y que se enmarca la tendencia a comprender la inclusión social desde la discusión sobre las posturas del pos-colonialismo, en relación con los debates internacionales y las agendas globales de educación. Se subraya que la educación en el contexto globalizado impone nuevas formas de colonialismo, contraponiendo los derechos morales individuales, frente a los derechos legales que no pueden garantizar la participación plena de todos. Las teorías poscoloniales proponen, entonces, reconsiderar el papel de quienes educan, además, llaman a un compromiso con la inclusión para fomentar prácticas educativas que desafíen y confronten la discriminación y la exclusión en todas sus formas (Lavia, 2007). Al respecto, la posición de la autora es que la inclusión educativa es indispensable para reposicionar la pedagogía también en los entornos universitarios, potenciadores de una responsabilidad social compartida garante de mejores oportunidades y de una mejor calidad de vida. 
URL: http://www.una.ac.cr/educare

\section{Conclusiones}

Desde la perspectiva socio-crítica se logra establecer una relación contextual entre las concepciones dominantes en la sociedad y las prácticas sociales vigentes en determinados momentos socio-históricos. En materia de discapacidad, este análisis posibilita ahondar en las dimensiones sociopolíticas e ideológicas, al develar las relaciones de poder subyacentes.

La superación de concepciones y formas de pensar pertenecientes a paradigmas ideológicos implica la ruptura de prácticas sociales excluyentes hacia distintos sectores de la población, arrastradas a lo largo de la historia. Dicha ruptura se hace necesaria para responder a las demandas sociales mundiales actuales, que apelan a la construcción de las identidades desde la participación, la convivencia y los derechos humanos y no, por el contrario, a la recurrente experiencia desde la marginación.

El enfoque socio-crítico adquiere relevancia, dado que posibilita el análisis de la dimensión política de la discapacidad que permea prácticas sociales e institucionales indispensables de cambiar para propiciar la inclusión educativa en la educación superior.

Se plantean, como consideraciones finales, las demandas de las tendencias actuales en educación superior en relación con la inclusión educativa, lo cual tiene implicaciones de cambio en las políticas institucionales y en las prácticas académicas tradicionales, con el propósito de generar nuevas formas de pensamiento que abran espacios a la diversidad desde un modelo educativo respetuoso de las diferencias y que permita la participación del colectivo con discapacidad en los distintos ámbitos institucionales.

\section{Referencias}

Apple, M. (1997). Teoría crítica y educación. Madrid: Miño y Dávila Editores.

Arnaiz, P. (mayo, 2010). ¿Cómo avanzar para que la educación inclusiva llegue a todos? Conferencia Inaugural presentada en el VI Encuentro Internacional de Inclusión Educativa. San José, Costa Rica.

Barnes, C. (1998). The Social Model of Disability: A Sociological Phenomenon Ignored by Sociologists? [El modelo social de discapacidad: Un fenómeno ignorado por sociólogos?]. En T. Shakespeare (Ed.), The Disability Reader: Social Science Perspectives [El lector de discapacidad: Perspectivas de ciencias sociales] (pp. 65-78). Londres: Continuum International Publishing Group.

Barnes, C. (2001). Disabled People in Britain and Discrimination. A Case for Anti-discrimination Legislation [Personas con discapacidad en Inglaterra y discriminación. Un caso para legislación antidiscriminatoria]. London: British Council of Organizations of Disabled People. 
Barnes, C. (enero, 2007a). Disability Studies: What's the Point [Estudios de discapacidad: Cuál es el punto]. Intersticios. Revista sociológica de pensamiento crítico, 1(1), 49-58. Recuperado de http://www.intersticios.es/article/view/673/549

Barnes, C. (noviembre, 2007b). Some thoughts on an inclusive Higher Education system. Developing and Embedding Inclusion: Policy and Practice in Higher Education. [Algunos pensamientos sobre un sistema inclusivo de educación superior. Desarrollando y abrazando la inclusión: Política y práctica en educación superior]. Conferencia 'Making Progress'. 12 de noviembre del 2010. Aston Bussiness School, Birmingham, Londres.

Bolt, D. (2004). Disability and the Rhetoric of Inclusive Higher Education [Discapacidad y la retórica de la educación inclusiva]. Journal of Further Higher Education, 28(4), 353-358.

Bourdieu, P. (1991). El sentido práctico. Madrid: Taurus.

Brogna, P. (2005). El derecho a la igualdad... ¿o el derecho a la diferencia? El Cotidiano, 21(134), 43-55. Recuperado de http://redalyc.uaemex.mx/pdf/325/32513407.pdf

Ferrante, C. (septiembre, 2007). Algunas reflexiones sobre la situación de las personas con discapacidad motora en la Argentina en la actualidad. Ponencia presentada en las IV Jornadas de jóvenes investigadores del Instituto de investigaciones Gino Germani. 19-20-21 de setiembre del 2007. Buenos Aires, Argentina. Recuperado de http://webiigg.sociales.uba. ar/iigg/jovenes investigadores/4jornadasjovenes/EJES/Eje\%207\%20Politicas\%20del\%20 Cuerpo/Ponencias/FERRANTE,\%20Carolina.pdf

Ferrante, C. y Ferreira, M. (2008). Cuerpo, discapacidad y trayectorias sociales: Dos estudios comparados. Revista de antropología experimental, 8(29), 403-428. Recuperado de http:// www.ujaen.es/huesped/rae/articulos2008/29ferrante08.pdf

Ferrante, C. y Ferreira, M. (2011). Cuerpo y habitus: El marco estructural de la experiencia de la discapacidad. Intersticios. Revista sociológica de pensamiento crítico, 5(2), 85-101. Recuperado de http://www.intersticios.es/article/view/8888

Ferreira, M. (octubre, 2007). Sociología de la discapacidad: Investigación y compromiso. Ponencia presentada en el III Congreso nacional discapacidad y universidad, Zaragoza. Recuperado de http://www.um.es/discatif/TEORIA/Ferreira Zaragoza.pdf

Ferreira, M. y Díaz, E. (octubre, 2007). La discapacidad: Una modalidad inexplorada de exclusión social. Ponencia presentada en el III Congreso nacional discapacidad y universidad. 24-26 de octubre del 2007. Zaragoza, España. Recuperado de http://www.um.es/discatif/TEORIA/ FerreiraDiaz Zaragoza.pdf

Ferreira, M. y Díaz, E. (2009). Discapacidad, exclusión social y tecnologías de la información. Política y Sociedad, $46(1$ y 2), 237-253. 
URL: http://www.una.ac.cr/educare

Finkelstein, V. (1980). Attitudes and Disabled People: Issues for Discussion [Actitudes y personas con discapacidad: Hechos para la discusión]. Washington, DC: World Rehabilitation Fund.

Hanafin, J., Shevlin, M., Kenny, M. y Mc Neela, E. (setiembre, 2007). Including Young People with Disabilities: Assessment Challenges in Higher Education [Incluyendo gente joven con discapacidad: Desafíos de la evaluación en la educación superior]. Higher Education, 54(3), 435-448. doi. 10.1007/s10734-006-9005-9

Lavia, J. (2007). Repositioning Pedagogies and Postcolonialism: Theories, Contradictions and Possibilities [Reposicionando pedagogías y poscolonialismo: Teorías, contradicciones y posibilidades]. International Journal of Inclusive Education, 11(3), 283-300. doi. 10.1080/13603110701237548

Manning, N. y Oliver, M. (1985). Madness, Epilepsy and Medicine [Locura, epilepsia y medicina]. En N. Manning (Ed.) Social Problems and Welfare Ideology [Problemas sociales e ideología del bienestar]. UK: Gower.

Martínez, J. (s. f.). Discapacidad:Evolución de conceptos. [Diapositivas en PowerPoint]. Recuperado de http://campus.usal.es/ lamemoriaparalizada/documentos/pdf/martinez perez.pdf

Miranda, G. (2004). Capitalismo global y cambio educativo. Los fundamentos estructurales e ideológicos de la política educativa neoliberal. Educare, (7), 13-42.

Moran, A. (mayo, 2007). Embracing Inclusive Teacher Education [Abrazando la educación inclusiva docente]. European Journal of Teacher Education, 30(2), 119-134.

Oliver, M. (1990). The Politics of Disablement. In Critical Texts in Social Work and the Welfare State [Las políticas de discapacidad. En textos críticos en trabajo social y el estado de bienestar]. Londres: MacMillan Press.

Oliver, M. (1996). Defining Impairment and Disability: Issues at Stake [Definiendo deficiencia y discapacidad: Hechos en juego]. En C. Barnes y G. Mercer (Eds.). Exploring the Divide; Illness and Disability [Explorando lo dividido: Enfermedad y discapacidad] (pp. 29-54). Leeds: The Disability Press.

Oliver, M. (diciembre, 2002). Emancipatory Research: A vehicle for Social Transformation or Policy Development [Investigación emancipatoria: Un vehículo para la transformación social o el desarrollo de políticas]. Trabajo presentado en el $1^{\text {st }}$ Annual Disability Research Seminar, Dublin. Recuperado de http://www.leeds.ac.uk/disability-studies/archiveuk/ Oliver/Mike's\%20paper.pdf

Popkewit, Th. (1997). La sociología política de la reforma educativa: Poder, saber y escolarización (2a. ed). En Th. Popkewit (Aut.), Sociología política de las reformas educativas. (pp. 25-57). Madrid: Ediciones Morata. 
Pumfrey, P. (febrero, 2008). Moving Towards Inclusión? The First-Degree Results of Students with and Without Disabilities in Higher Education in the UK: 1998-2005 [¿Moviéndose hacia la inclusión? Los resultados de primer grado de los estudiantes con discapacidad y sin esta en la Educación Superior en el Reino Unido: 1998-2005]. European Journal of Special Needs Education, 23(1), 31-46.

Riutort, B. (2001). De la regulación a la globalización. En B. Riutort (Ed.). Razón, política, globalización y modernidad compleja (pp. 15-54). Barcelona: El viejo Topo.

Shakespeare, T. (1993). Disabled People's Self Organization: A New Social Movement? [Organización propia de las personas con discapacidad: ¿Un nuevo movimiento social?]. Disability, Handicap and society, 8(3), 249-264.

Sheeran, Y., Brown, B. y Baker, S. (mayo, 2007). Conflicting Philosophies of Inclusión: The Contestation of Knowledge in Widening Participation [Filosofías en conflicto sobre inclusión: La respuesta del conocimiento en la ampliación de la participación]. London Review of Education, 5(3), 249-263.

Skelton, A. (abril, 2002). Towards Inclusive Learning Environments in Higher Education? Reflections on a Professional Development Course for University Lecturers [¿Hacia ambientes de aprendizaje inclusivos en la educación superior? Reflexiones sobre un curso de desarrollo profesional universitario]. Teaching in Higher Education, 7(2), 193-214.

Sojo, C. (2010). Igualiticos: La construcción social de la desigualdad en Costa Rica. San José, Costa Rica: Master Litho/PNUD. Recuperado de http://www.enlaceacademico.org/uploads/ media/IGUALITICOS Sojo 2010 02.pdf

Williams, D. A. (verano, 2006). Inclusive Excellence: UConn Builds Capacity for Diversity and Change [Excelencia inclusiva: Universidad de Connecticut construye capacidad para la diversidad y el cambio]. The Journal of the New England Board of Higher Education, 21(1), 17-19. 